\title{
Reflexividad sobre la intervención profesional en duelo con población afectada por el conflicto armado en Colombia
}

\author{
María Cénide Escobar-Serrano \\ Trabajadora Social. Especialista en Intervención con Familias \\ Universidad del Valle. Cali, Colombia \\ https:/ / orcid.org/0000-0002-0476-0715 • maria.escobar@correounivalle.edu.co
}

\author{
Maritza Charry-Higuera \\ Trabajadora Social. Magíster en Intervención Social \\ Universidad del Valle. Cali, Colombia \\ https:// orcid.org/0000-0002-1967-2750 • maritza.charry@correounivalle.edu.co
}

\section{Natalia Ramírez-Moncada \\ Trabajadora Social \\ Fundación Cultiva el Arte y la Cultura. Bogotá, Colombia \\ https:// orcid.org/0000-0001-6795-080X • natalia.moncada@correounivalle.edu.co}

\section{Resumen}

El presente artículo contiene los resultados de la investigación que buscó reflexionar sobre la intervención para develar comprensiones del duelo y provocar procesos autorreferenciales con los profesionales que atienden población afectada por el conflicto armado colombiano. Se aplicó una metodología cualitativa desde una epistemología liminal que concibe la existencia de límites difusos con la intervención; se realizaron entrevistas individuales y encuentros grupales que favorecieron la reflexividad entre los profesionales. Los resultados muestran preocupación de las profesionales por los alcances institucionales de la intervención en duelo, evidencian vacíos en su formación e historia de duelos inconclusos que les impide implicarse y profundizar en la intervención en duelo con la población. Se concluye que la reflexividad frente a la intervención en duelo conlleva repensar lo que hacen y cómo lo hacen: destacar limitaciones y alcances de los contextos institucionales, mayor profundidad en la intervención, formarse en torno al duelo y prepararse emocionalmente acerca de sus propias pérdidas para generar relaciones profesionales que faciliten procesos de elaboración y reconstrucción de las situaciones dolorosas que viven las personas que atienden.

Palabras clave: Duelo; Intervención; Reflexividad; Trabajo Social; Conflicto armado en Colombia.

Recibido: 01/10/2019 | Aprobado: 14/11/2019

(i)(-)(2) Esta obra está bajo una Licencia Creative Commons Atribución-NoComercial-CompartirIgual $\underline{4.0 \text { Internacional. }}$

Procedencia del artículo: Artículo producto de la investigación Reflexividad sobre la intervención en duelo de trabajadores/as sociales con mujeres y hombres en situación de desplazamiento forzado. Código 395, financiado por la Vicerrectoría de Investigaciones de la Universidad del Valle. Cali, Colombia.

\section{¿Cómo citar este artículo? / How to quote this article?}

Escobar-Serrano, M.C., Charry-Higuera, M., y Ramírez-Moncada, N. (2020). Reflexividad sobre la intervención profesional en duelo con población afectada por el conflicto armado en Colombia. Prospectiva. Revista de Trabajo Social e intervención social, (30), 67-89. doi: 10.25100/prts.v0i30.8564. 
Escobar-Serrano, Charry-Higuera, y Ramírez-Moncada

\title{
Reflexivity about professional intervention bereavement with population affected by the armed conflict in Colombia
}

\begin{abstract}
This article contains the partial results of the ongoing investigation that sought to reflect on the intervention, reveal understandings of grief and provoke self-referential processes with the professionals who serve the population affected by the Colombian armed conflict. The methodology was qualitative from a liminal epistemology that conceives the existence of diffuse limits with the intervention; individual interviews and group meetings were found that favored reflexivity among professionals. The results affected concern professionals for the institutional progress of the bereavement intervention, gaps in their training and history of unfinished grief that prevent them and deepen the bereavement intervention with the population. It is concluded that reflexivity in the face of grieving intervention implies rethinking what they do and how they do it: highlighting limitations and scope of institutional contexts, greater depth in the intervention, training around grief and preparing emotionally about their own losses to generate professional relationships that facilitate the elaboration and reconstruction processes of the painful situations experienced by the people they serve.

Keywords: Grief; Intervention; Reflexivity; Social Work; Colombian armed conflict.

Sumario: 1. Introducción, 2. Metodología, 3. Hallazgos, 3.1 Alcance de la intervención en duelo, 3.2 Comprensión de los profesionales sobre el duelo y su formación, 3.3 Historia de pérdidas del profesional y la relación con la intervención en duelo, 4. Conclusiones, 5. Referencias bibliográficas.
\end{abstract}




\section{Introducción}

En el marco del conflicto armado en Colombia la intervención profesional viene copando las agendas de investigadores de distintas disciplinas, entre ellas Trabajo Social y Psicología. La intervención busca no sólo comprender, sino responder a las consecuencias que viven las personas afectadas por el conflicto armado.

La ley de Víctimas 1448 del 2011 constituye un avance importante por parte del Estado Colombiano porque ha reconocido a las Víctimas en todas sus dimensiones y las ha integrado a un sistema de participación. Asimismo, se han creado instituciones y programas que garanticen los derechos de las víctimas y la rehabilitación, entendida como una medida de reparación integral. El Protocolo de Atención Integral en Salud con Enfoque Psicosocial a Víctimas del Conflicto Armado (PAPSIVI) es el componente más importante de esta medida. Sin embargo, los esfuerzos y las estrategias de intervención implementadas por los profesionales resultan insuficientes, como lo refieren diversos estudios de organismos internacionales e investigaciones nacionales ${ }^{1}$, que han develado tardanza en la atención, delimitación temporal no coincidente con las necesidades psicológicas de la población y falta de experiencia de los profesionales en la intervención con víctimas, (Portilla y Correa, 2015). Igualmente, resalta este último estudio que las víctimas reiteran:

La necesidad de contar con asistencia psicosocial y otras formas para superar el duelo, porque lo recibido por parte del Estado en materia de atención psicológica ha sido poco útil y deficitario y en algunos casos, aunque han recibido medicación, no han recibido la atención y seguimiento adecuado en salud mental, pero no la que le ofrecen a cualquier paciente psiquiátrico sino medidas específicas para la elaboración y la superación del trauma. (p. 51).

La población atendida manifiesta que el acompañamiento no es sistemático, ni planeado de manera diferencial para entender la subjetividad de las víctimas, en especial de niños, niñas y adolescentes (Chávez y Bohórquez, 2011). Algunas prácticas

1 El informe de Portilla y Correa (2015) presenta una aproximación al estado de avance de la implementación de la política de reparación individual de la Ley de Víctimas, a partir de un ejercicio de entrevistas y grupos focales con funcionarios nacionales y regionales de la Uariv y otras entidades del Snariv (Sistema Nacional de Atención y Reparación Integral a Víctimas).También se contactó a víctimas de homicidio, desaparición forzada y violencia sexual. Se buscó establecer la diferencia entre el diseño formal y la aplicación práctica de la reparación a víctimas. El informe hace la salvedad de que se trata de un proceso particularmente complejo que requiere la intervención de numerosos organismos y entidades del Estado, por tanto, es aún temprano para realizar afirmaciones concluyentes acerca de las fases del proceso y su estado de avance. Otro documento que da cuenta de los avances de la implementación de la Ley 1448 de 2011 es el VIII informe sobre los avances de la Política de atención, asistencia y reparación integral a Víctimas del conflicto, entregado por el Gobierno Colombiano en el 2018. 
Escobar-Serrano, Charry-Higuera, y Ramírez-Moncada

institucionales ejercen violencia simbólica sobre la población al no reconocer sus intereses, recursos y demandas (CICR, 2016; Vargas, 2009). Las víctimas no se sienten escuchadas, ni tratadas de manera digna y expresan haber recibido un trato "hostil y grosero"; perciben que los profesionales tienen poca formación y sensibilidad frente al "sufrimiento y el dolor" (CICR, 2016, pp. 23-28) lo que hace que dediquen poco espacio a escuchar y abordar el dolor (García, 2013); es decir, sienten que no reconocen su dolor y se las revictimiza (Meza, 2012; Rodríguez, 2017), lo cual dificulta su recuperación y/o asimilación de las nuevas realidades; tampoco obtienen respuestas respecto de los mecanismos, procedimientos y sistemas de información (CICR, 2016).

La violencia sociopolítica ha sido un tema denso que moviliza el interés de investigadores ${ }^{2}$ debido a los múltiples efectos ${ }^{3}$ que ha traído el conflicto armado en los últimos 70 años en la población colombiana. Los entornos disruptivos creados producen implicaciones subjetivas y pérdidas ocasionadas por los distintos hechos victimizantes, tipificados jurídicamente en Colombia4. Entre los efectos del conflicto armado tan intenso y duradero vivido en el País, los investigadores resaltan la elaboración de acontecimientos traumáticos (Munévar y Castro, 2018); miedo, pena, rabia y desprotección (Carbonell et al., 2015; Peláez, 2007); pluralidad de pérdidas y duelos (Díaz, Molina y Marín, 2015; Rodríguez, 2017); impactos en el proceso de adaptación de los niños al sistema escolar (Lasso, 2013) y dificultades de las mujeres para tramitar el abuso sexual (Betancourt, 2011), entre otros, que remiten a la vulnerabilidad por género y ciclo vital de estos grupos poblacionales.

En definitiva, son los profesionales quienes deben trabajar las enormes cuotas de sufrimiento psicológico, enfermedad psíquica y catástrofe social (Benyakar, 2016), así como facilitar la elaboración de los duelos de las víctimas. Sin embargo, estos duelos se han venido enconando históricamente (Peláez, 2007) por el desconocimiento de los daños subjetivos, del dolor y de las reparaciones incompletas que no permiten la reconstrucción

2 El estudio de Guzmán, Fals-Borda y Umaña (1962) es un punto de referencia entre los prolíficos estudios sobre la violencia en Colombia. González (2014), ha trabajado en sentido histórico y geográfico la violencia en Colombia y Cancimance-López (2013; 2015), trabajador social, ha procurado una comprensión de la violencia, conectada con procesos de resistencia de las comunidades para afrontar el dolor y sobrevivir.

3 A octubre 1 del 2018, se habían registrado 8.760.290 víctimas, de las cuales 8.400 .856 corresponden al conflicto armado; de ellas 6.977.066 fueron sujetos de atención (Registro Único de Víctimas en Colombia, 2018). La ENSM señala que el conflicto armado en Colombia ha causado 220.000 muertes de personas, desde 1958 hasta diciembre de 2012, "aunque el costo humano de los duelos y el dolor no puede ser valorado, se calcula que en América Latina el 14.2\% del producto interno bruto (PIB) se invierte en atender los costos directos, indirectos o por transferencia de la violencia".

4 Abandono o despojo forzado de tierras; acto terrorista/atentados/combates/hostigamientos; amenaza; confinamiento; delitos contra la libertad y la integridad sexual; desaparición forzada; desplazamiento; homicidio; lesiones personales físicas; lesiones personales psicológicas; minas antipersonas/munición sin explotar/artefacto explosivo; pérdida de bienes muebles o inmuebles; secuestro; tortura y vinculación de niños, niñas y adolescentes (Unidad Nacional de Víctimas). 
Escobar-Serrano, Charry-Higuera, y Ramírez-Moncada

del sentido del orden y justicia en las víctimas (Díaz, 2012). Cuando los duelos no se sanan se aumentan las repercusiones individuales y sociales, favoreciendo la reproducción de la violencia (Díaz et al., 2015):

Así como la no elaboración a nivel individual puede tener repercusiones sociales, psicológicas y emocionales, a nivel grupal las consecuencias pueden alimentar angustias, impotencias o rabias colectivas que al carecer de medios de expresión terminan o bien en la memoria repetitiva y no procesada de la venganza, en la internalización del odio, en la construcción de ideologías de exterminio, o bien en la autodestrucción. (Riaño, 1998, p. 104).

En razón a la complejidad sociopolítica que atraviesa Colombia, sobre la intervención de los profesionales que trabajan en los organismos del Estado recae gran parte del peso de la reparación, del cambio y de la adaptación de la población, desconociéndose aspectos estructurales que favorecen las incongruencias entre la norma, la acción de las políticas públicas ${ }^{5}$ y el asistencialismo (Cancimance-López, 2013; Torres, 2011). En muchos programas prima el modelo psiquiátrico en el que la dimensión emocional y cultural es secundaria (Munévar y Castro, 2018), omitiendo las particularidades de la vivencia de los duelos; en otros estudios los profesionales señalan como preocupante la intervención inoportuna y fragmentada (Portilla y Correa, 2015; Rodríguez, 2017), el establecimiento de vínculos fugaces y utilitaristas entre profesionalescomunidades, limitaciones que, según Vargas (2009) no contribuyen a la elaboración de las realidades con la población.

Los(as) trabajadores sociales y psicólogos que intervienen con población afectada por el conflicto armado han venido reflexionando y configurando su acción profesional como objeto de conocimiento (Bello, 2009; Casillas, 2018; Fombuena, 2011; Mosquera, 2006; Velázquez 2012). Esto es lógico, por cuanto para el Trabajo Social la intervención se considera como una construcción que corresponde a un entramado de relaciones que suscita un intercambio entre los sujetos y los profesionales que los atienden (Mosquera, 2012; Vargas, 2009) e implica la reflexión del profesional sobre sus posturas (Moreno y Díaz, 2016). Si bien, la intervención profesional en duelo involucra la revisión del profesional acerca de su historia de pérdidas a lo largo del ciclo vital (Chaurand, Feixas y Neimeyer, 2010), este ejercicio no es tan frecuente en los profesionales que intervienen en duelo; por ello se buscó con la investigación un encuentro con los profesionales para favorecer la reflexión sobre la intervención, develar sus comprensiones sobre el duelo y provocar procesos autorreferenciales que beneficiaran el reconocimiento de su historia de pérdidas y su lugar en el ejercicio profesional. En este artículo se alude a tres temas

5 En contextos de alta conflictividad, una reparación integral requiere de la articulación Estado y sociedad en un compromiso con la verdad, la justicia y la reparación de las víctimas, condiciones indispensables para tramitar los duelos individuales y colectivos, (Díaz, 2012). 
Escobar-Serrano, Charry-Higuera, y Ramírez-Moncada

movilizados durante las conversaciones reflexivas entre investigadores y profesionales en espacios individuales y grupales durante los meses de febrero a julio de 2019: alcance y descripción de la intervención en duelo, comprensión y formación sobre el duelo, e historia de pérdidas del profesional.

\section{Metodología}

La metodología de la investigación fue de orden cualitativo y se enmarcó en aquellos movimientos epistemológicos en los que el conocimiento no puede darse por sentado, ni como una verdad absoluta (Páramo, 2013), sino como construcciones sobre la realidad y la intervención entre los distintos actores. La epistemología liminal, que según Torres (2004) concibe la existencia de límites difusos entre la investigación y la intervención, no es reciente en el trabajo del Grupo de Investigación Estudios de Familia y Sociedad6. En la lógica de esta perspectiva epistemológica, acogimos una comprensión compleja del duelo que integra la fundamentación psicoanalítica y los aportes del constructivismo en torno al duelo y su intervención, como marco propicio para la reflexividad a través de conversaciones acerca de la intervención con los profesionales.

Comprendemos que las manifestaciones de dolor provenientes de la multiplicidad de pérdidas que viven las personas en contextos de violencia son reguladas por el repertorio cultural y tienen lugar al mismo tiempo en tres sistemas: el sí mismo, la familia y la sociedad. En el sí mismo y la familia, el duelo es un proceso activo que empuja a los supervivientes a la toma de decisiones concretas (conscientes o inconscientes), de reconstrucción de la experiencia de pérdida a través del intercambio de las subjetividades interconectadas con el entorno social y cultural (Neimeyer, 2002; Ospina, 2014). Para su elaboración, es necesario que se establezcan procesos de comunicación con los otros que movilicen el dolor, el reencuentro y la recuperación de la confianza en los vínculos; éstos pueden establecerse con allegados y/o profesionales cuando el acuerdo social de expresión del dolor se altera. Justamente, a nivel social, se habla del duelo colectivo como una categoría que surge de las contradicciones de la situación social y de sufrimiento éticopolítico de las comunidades latinoamericanas, y se reconoce como aquel que registra el padecimiento que viven las personas y las comunidades ante las situaciones de guerra como producto de procesos sociales y políticos que para su elaboración requiere la creación de órdenes sociales más justos, diferentes de aquellos en los que se generó la violencia (Di Maio, 2015; Uribe, 2008).

6 Los primeros estudios acerca de la intervención e investigación en procesos de duelo son de la profesora Ana María Ospina Velasco (QEPD), pionera del campo de conocimiento del Trabajo Social y la Tanatología. Sus trabajos fortalecieron una comprensión compleja de la muerte, las pérdidas y el dolor de los sobrevivientes que afrontan distintos tipos de duelos. Sus desarrollos se articulan con el Psicoanálisis, campo en el cual fundamentó la comprensión del dolor humano, la relación profesional y la formación de trabajadores/as sociales (Ospina, 2014). 
Escobar-Serrano, Charry-Higuera, y Ramírez-Moncada

No considerar presupuestos de objetividad ni explicaciones totalizantes - porque de acuerdo con Ibáñez (1994, p. XI) “el sujeto no está separado del objeto, y en la investigación del objeto quedan necesariamente huellas del sujeto, porque el objeto es producto de la actividad objetivadora del sujeto" - es acorde con la intención metodológica de generar reflexión colectiva acerca de las comprensiones y la intervención en duelo entre las investigadoras y las/os profesionales; de allí que a lo largo del artículo aparecen entrelazados antecedentes y referentes teóricos sobre el duelo, pero también ideas, pensamientos y sentimientos de las profesionales sobre el mismo7.

En este sentido, la investigación fue de segundo orden, al intentar tejer la perspectiva de las investigadoras y de las participantes como dos representaciones de la realidad plural, diversa y parcial (Bourdieu y Wacquant, 2005); así como cuando se convocaba a volver sobre las explicaciones y descripciones dadas y se hace el llamado a revisar las formas de describir, contrastar y conversar de manera reflexiva acerca de la realidad (Garzón, 2008).

Según Zemelman, citado por Torres y Torres (2000), las reflexiones articulan lo dado y lo posible, la memoria y el futuro, la historia y la política; permiten comprender desde la perspectiva de la subjetividad de los sujetos sociales, porque ellos y ellas conforman un horizonte en el que confluyen los diferentes planos de la realidad social ${ }^{8}$. La reflexividad remite a un sujeto que construye sentidos a sus trayectorias, que, en la segunda modernidad, corresponden a una nueva relación entre la sociedad y el individuo, en el que éste es agente de su biografía (Dubet, 2010).

Asumir el paradigma reflexivo en investigación en Trabajo Social, implicó una articulación teórica y metodológica que, según Montagud (2015), reúne las categorías reflexividad y complejidad.

Desde un punto de vista antropológico la investigación no puede evitar el compromiso político con los asuntos que investiga, debe reflexionar para establecer una relación entre el conocimiento académico y el conocimiento práctico y político de lo que

7 El duelo se inscribe en una línea de investigación que articula la intervención en el Grupo Estudios de Familia y Sociedad. Por esto, proponer investigar el tema, surge de la inquietud por conocer cómo definen, comprenden y abordan los profesionales el duelo. Esta categoría evidenció en su exploración la amplitud de estudios que sobre el particular se han realizado desde diferentes perspectivas disciplinares, en las que el psicoanálisis, con el estudio de Freud sobre melancolía y duelo, es referente obligado.

8 La "racionalidad epistémica", propuesta por Zemelman, implica un esfuerzo crítico por razonar sobre "los determinismos históricos y valóricos en los que está inmerso el mismo investigador, así como su objeto de estudio" (p. 4). La subjetividad es singular e histórica, se hace y deshace, puede ser transitoria o permanente, se construye en la medida en que el sujeto amplía su conciencia histórica y "forja ámbitos de sentido en cuyos cauces hay que ver las conductas y las experiencias, en forma de responder al desafío de transformación" (Torres y Torres, 2000, p. 5). 
Escobar-Serrano, Charry-Higuera, y Ramírez-Moncada

investiga (Gledhill, 2000). De esta forma, asumiendo en algún sentido el compromiso político del Trabajo Social con los procesos de sufrimiento humano y de justicia social, se indagó a partir del "análisis de los contextos, los usos, los significados y los efectos de las intervenciones concretas", buscando con ello, además, hacernos conscientes de ciertas relaciones de poder propias de la sociedad contemporánea que limitan la comprensión de lo humano, el manejo político de los acontecimientos y las maneras de enfrentar el cambio y las transformaciones sociales (García y Ruendeles, 2017, p. 244).

Las técnicas e instrumentos elegidos buscaron activar conversaciones entre las participantes posibilitando ampliar campos de reflexión sobre las propias prácticas y la forma en que se nombra y llena de contenidos, emociones, sentimientos y pensamientos la realidad. La reflexión, en sentido complejo requiere de dos movimientos, en uno prevalece la cercanía y en el otro la distancia; el primero privilegia la descripción y la explicación, mientras que en el otro se propicia la observación de las operaciones mediante las cuales llegamos a la descripción (Sánchez y Escobar, 2009, pp. 49-53; 333). Las técnicas seleccionadas fueron: las entrevistas, que permitieron reconocer lo particular de las experiencias; y los encuentros grupales, que procuraron favorecer la participación, la interacción y conversaciones reflexivas entre las asistentes. Con ambas técnicas, se trató de trascender la recolección de información y ubicarse más bien en el terreno de lo vinculante, como formas de conocernos, reconocernos en nuestras acciones, pensamientos, sentimientos, activando la interpretación y la reflexión respecto a la intervención en duelo y la revisión de sus historias de pérdidas. Intentamos construir un vínculo con las Trabajadoras Sociales que trabajan en el PAPSIVI - la ESE Oriente en Cali, que voluntariamente aceptaron participar del proceso de investigación.

\section{Hallazgos}

\subsection{Alcance de la intervención en duelo}

El alcance de la intervención en duelo con víctimas del conflicto armado que realizan las profesionales como parte del Equipo Psicosocial del Programa PAPSIVI, estuvo circunscrito, de un lado a las orientaciones y lineamientos que éste plantea, y del otro, a las reflexiones que dichas profesionales hicieron en torno a las interferencias e insuficiencias que encuentran en la implementación, ambas estrechamente relacionadas. Sobre el primer aspecto, el trabajo de investigación-intervención adelantado con las profesionales estuvo signado por una idea recurrente de que ellas no hacían intervención, sino atención psicosocial acorde a lo que establece la Ley 1448 de 2011. La distinción que ellas hacen entre atención e intervención la sustentan en las Orientaciones Metodológicas para la atención individual y familiar contempladas en los volúmenes 2 y $3^{9}$, en los que se definen dos

9 Oficina de Promoción Social (2018a; 2018b). 
Escobar-Serrano, Charry-Higuera, y Ramírez-Moncada

formas particulares de atención: la atención en consejería/apoyo y la atención terapéutica. De igual manera, según dichas orientaciones la valoración que hagan las profesionales frente a la severidad y complejidad de los impactos define las formas de atención psicosocial individual y familiar que requieren las víctimas.

El reconocimiento que hace el Estado de que, en su mayoría, las personas afectadas por el conflicto armado experimentan duelos complicados y que requieren una atención terapéutica es lo que las profesionales denominan intervención. Es decir, reconocen la intervención desde un punto de vista clínico/terapéutico más que social. Esta distinción tiene que ver, entre otras cosas, con una tendencia de tradición epistémica que separa individuo y sociedad, que no en pocas oportunidades en el terreno del actuar profesional e institucional se resuelve como "atención psicosocial", develando no solo un contenido de las acciones, sino la relación entre lo psíquico y lo social y el lugar que a ésta se le da. Valdría la pena que el Programa PAPSIVI volviera sobre esta discusión epistemológica, porque no es un problema de orientación técnica, sino una discusión de vieja data entre diversas disciplinas preocupadas por los impactos políticos de la intervención y sus efectos sobre las víctimas ${ }^{10}$.

Como se puede ver, la intervención es una categoría conceptual compleja por su carga polisémica que, para el Trabajo Social, está estrechamente relacionada con los interrogantes: cuándo, para qué, con quién y cómo favorecer transformaciones, lo cual tiene que ver con las dimensiones sociales, políticas, éticas y culturales. En el caso de la intervención en duelo, la entendemos como un proceso de construcción social entre los actores y una forma de colaboración para la reconstrucción de los significados, acerca de las pérdidas que favorecen la reorganización de la vida y las actuaciones de las personas (Ospina, 2014). Se resalta la importancia de reconocer a las personas con quienes se trabaja como pares epistémicos, para dialogar y co-construir saberes, asumiendo una actitud de horizontalidad, en la que la relación entre los actores tiene un lugar preponderante (Mosquera, 2012).

Para contribuir a la elaboración de los duelos, los y las profesionales deben establecer procesos de interacción y comunicación con las víctimas que movilicen el dolor, el reencuentro y la recuperación de la confianza en los vínculos. No obstante, en la vida cotidiana, los lineamientos del PAPSIVI interfieren con su accionar profesional y limitan el alcance de la intervención. Es decir, la división conceptual entre la recuperación y mitigación de los daños y el sufrimiento (a cargo del Equipo de Atención Psicosocial, que hizo parte de la investigación) y la rehabilitación de la población víctima del conflicto armado (a cargo del Equipo de Atención Integral en Salud con Enfoque Psicosocial), se cumple

10 La fundamentación epistémica sobre la diferencia entre atención psicosocial y la intervención pueden consultarse en Moreno y Moncayo (2015). 
Escobar-Serrano, Charry-Higuera, y Ramírez-Moncada

procedimentalmente, y al mismo tiempo arrasa con la posibilidad de intervenir de manera más profunda en las necesidades de elaboración de los duelos de las personas y sus familias. Ante la tensión evidenciada, las profesionales se preocupan por profundizar el abordaje del dolor en la intervención profesional:

El tema de duelo es algo muy complicado porque estamos trabajando directamente con los sentimientos de las personas, entonces no es llegar y hacer una actividad, es saber que esa actividad va a llegar directamente a ella, y que va a generar un cambio o al menos una tranquilidad, porque si identificamos que en esa persona hay que trabajar duelo es porque hay un dolor, entonces hay que saber de qué forma vamos a mitigar el dolor, no es llegar, doña llegué, venga hagamos esto y listo, salió, es saber de qué forma hacerlo, con corazón y tener el sentido de transformación ante la persona. (E11: Trabajadora social).

Hay una orientación metodológica que tenemos como referencia para la atención y hay una familiar, que tiene que ver con procesos de duelo, pero cuando uno revisa esas actividades, las técnicas que se proponen están más enfocadas al tema de la pérdida, por quién ya no está físicamente... pero es solo una orientación. (E16: Trabajadora social).

Las profesionales conocen y desarrollan los procedimientos del PAPSIVI, identifican necesidades de la población, la situación a intervenir, la gravedad del daño, definen un plan de trabajo, la modalidad de atención y que las personas estén interesadas y accedan; evidenciando así un conocimiento "técnico", los fundamentos metodológicos, las modalidades y los momentos del Programa. El inicio del proceso de intervención se caracteriza por la posibilidad de que las personas cumplan con los requisitos del filtro, entre ellos que hayan sido catalogadas jurídicamente como víctimas. Cuando las personas acceden a la atención es cuando se materializa la intervención y se apacigua el ambiente de incertidumbre por el cumplimiento de las metas: "Nosotros trabajamos por meta, son ochenta personas que debemos atender cada dos meses, se trabajan ocho sesiones..." (E11: Trabajadora social).

Para los casos de atención individual y familiar se propone un tope de ocho sesiones, esta delimitación de sesiones ha confrontado a las profesionales, quienes consideran que este tiempo es insuficiente para hacer procesos de intervención en duelos ocasionados por pérdidas en el conflicto armado ${ }^{11}$.

Como se mencionó, la distinción entre reparación y mitigación es viable en el plano operativo, pero es inviable en el plano relacional, porque para el profesional es casi

11 El estudio de Portilla y Correa (2015, p. 48) refiere un enfoque lineal para entender y atender la rehabilitación por su delimitación en el tiempo, porque "otras experiencias de atención psicosocial a víctimas de violaciones masivas a los derechos humanos consisten precisamente en programas de larga duración que ofrecen atención a víctimas en el momento en que ellas lo requieran". 
Escobar-Serrano, Charry-Higuera, y Ramírez-Moncada

imposible mitigar el dolor, entendida la mitigación como "atenuar o suavizar" el dolor del otro. Por el contrario, el profesional está formado para construir una relación de ayuda, que, en el contexto de la reparación, implica construir confianza para el reconocimiento del dolor del otro:

Las tres veces que he trabajado con procesos de duelo me han quedado como muchas dudas. Porque yo considero que los veo cada ocho días por dos meses, serían ocho sesiones, la primera, casi siempre es identificar en muy poco tiempo cuáles son las necesidades de la familia, en el segundo ya es como generar esa confianza, entonces uno genera la confianza con la familia y más en unos temas de esos, entonces lo que uno logra empezar a trabajar con la familia a veces se hace muy corto o uno empieza, termina renovando, o sea, sacando muchas cosas de la familia y uno ya está próximo a terminar de ver esa familia... (...) en ese afán de poder cumplir esas ocho sesiones a uno a veces le toca que omitir algunas cosas, saltarse a la otra, y al final me queda esa duda, ¿será que hice bien? ¿O lo que hice fue un daño? La forma como se interviene, creería yo que no es tanto la adecuada y que por más que uno trabaje uno logra movilizar a las personas desde su dolor, pero aún siento que hay elementos muy importantes para trabajar, porque por lo menos si son parte del conflicto armado uno queda con muchas preguntas... hay mucha rabia, dolor, y las respuestas no las va dando ni el tiempo, ni la justicia... siempre va a haber cosas que no lo vamos a lograr resolver. Muchas veces me doy cuenta de que ninguno de los temas de la guía me sirven, entonces yo empiezo a trabajar otros temas y luego los ajusto a algo de algún tema de la guía para poder argumentarlo. (E10: Trabajadora social).

El tema de que normalmente las instituciones ahora mecanizan al trabajador social, no dejan que tenga libertad para poder intervenir, simplemente nos limitan, vaya haga una atención y nos dan las pautas, y muchas veces la familia requiere más de lo que dicen las pautas, requiere mayor tiempo mayor compromiso por parte del profesional, y no se tiene en cuenta eso. (F11: Trabajadora social).

Tanto los profesionales como algunos autores señalan los retos que deben enfrentar los profesionales frente a las demandas institucionales, a los obstáculos de las políticas, a las lógicas institucionales de estandarización de los procesos (Blandón, Durango y Palacio, 2017; Gómez y Rodríguez, 2017; Munévar y Castro, 2018) y las limitaciones de tiempo y formación que no permiten dar continuidad y profundidad a la intervención en duelo. Estas limitaciones muestran el reconocimiento parcial de dimensiones conectadas. Una, referida a la institucional-normativa, y otra, a la personal, asociada con la formación en duelo, la calidad del vínculo y la relación establecida entre profesionales-población.

\subsection{Comprensión de los profesionales sobre el duelo y su formación}

La reflexión sobre la formación y la comprensión del duelo surgió alrededor de los casos más significativos que han atendido las profesionales durante su experiencia de trabajo en el PAPSIVI. Las profesionales coincidieron en que la formación universitaria 
Escobar-Serrano, Charry-Higuera, y Ramírez-Moncada

brinda herramientas básicas alrededor del tema del duelo por ser carreras que se ubican en áreas humanas; sin embargo, coinciden en la poca formación específica en duelo que no logra responder a las exigencias de intervención en los contextos de violencia política, en los que las personas están sometidas a eventos traumáticos que arrasan con su cotidianidad y sobrepasan su capacidad de cuidado y atención:

Lo que yo sabía era, digamos, con la formación académica. (EG8).

Cuando yo estaba en la universidad nosotros no alcanzamos a ver esta parte de muerte, nunca me dio por ponerme a leer nada acerca del tema. (F10: Trabajadora social).

Cuando estoy trabajando el tema se me generan una cantidad de dudas y a veces yo me meto a buscar... pero pues siento que todavía me queda como faltando ciertos elementos para poder trabajar ese tema. Uno lee en internet actividades o ciertas cosas que nos pueden guiar un poco. (E11: Trabajadora social).

El vacío en la formación universitaria frente al tema de la muerte y el duelo se evidencia ante la necesidad de intervenir con las familias que están atravesando duelos por pérdidas ocasionadas por el conflicto armado; cómo entender el duelo y cómo ayudar a las familias sin ocasionarles más daño es lo que lleva a las profesionales a interesarse, a leer acerca del tema del duelo en la internet, ya que los lineamientos del PAPSIVI son insuficientes:

Cuando yo ya me enfrento a mi vida laboral y me doy cuenta que me toca atender ese tema al principio decía no, ese tema yo no lo voy a trabajar, no eso es muy duro, o sea, empecé a indagar con las compañeras y ellas decían, cuando es tema de duelo yo prefiero no meterme por ahí, pero ya llegó un momento, entonces pues me va a tocar trabajar duelo, empecé a trabajar y me vi en la obligación de empezar a leer ciertas cosas para poder atender, porque la guía se quedaba corta, con esas ganas de no ser de las que hace acción con daño. (F10: Trabajadora social).

La formación para la intervención en duelo es un camino en construcción para el Trabajo Social de la Universidad del Valle, Programa del que todas las profesionales que participaron de la investigación son egresadas; pues si bien hay una trayectoria en el tema de duelo, no todos/as los/as estudiantes logran formarse sobre el particular, porque los contenidos curriculares sobre la pérdida, la muerte, el duelo y la orientación en duelo se recogen en asignaturas de carácter electivo ${ }^{12}$. Es preciso avanzar y fortalecer la formación

12 La asignatura electiva Muerte, morir y duelo fue creada por la profesora Ospina en el año 1992 para el Programa de Trabajo Social, y años más tarde para los Programas de Posgrados. Esta asignatura se focaliza en los procesos de pérdidas y duelos por enfermedad desde una perspectiva psicoanalítica y constructivista. Posteriormente, la profesora Charry en el año 2014 crea una segunda electiva, Muerte y duelo en contextos de violencia, que busca además de retomar la fundamentación en pérdidas y duelo proporcionada por la profesora Ospina, abordar la especificidad del dolor, las pérdidas y duelos generados por los contextos violentos del conflicto armado en Colombia. En el equipo de docentes del Grupo de Investigación Estudios de Familia y Sociedad existe el interés de mantener esta línea de trabajo 
sobre el tema del duelo, sobre todo porque vivimos en una sociedad que ha sido severamente golpeada por la pérdida y los horrores de la violencia socio-política, en tal sentido trabajos como los desarrollados por estudiantes de otros programas académicos, entre ellos de Sociología y Psicología, cobran relevancia, porque amplían la complejidad social y subjetiva del duelo. De igual manera, miradas críticas aportadas por los estudios de violencia política con perspectiva de género, son importantes porque aportan un enfoque crítico-político a la comprensión de las víctimas de la violencia, para que estas sean entendidas y asumidas socialmente.

El reconocimiento que le otorgan las profesionales a la importancia de la teoría ${ }^{13}$ se posibilita por el intercambio teoría-práctica; ante la realidad de las familias surgen cuestionamientos por el tema de las pérdidas y el duelo, pero consideran que no cuentan con los soportes conceptuales para hacerlo:

Llegó un momento, unas familias que efectivamente me tocaban, por más que le metía canalización de emociones no me daba, entonces pues me va a tocar trabajar duelo, empecé a trabajar con ellas y me vi en la obligación de empezar a leer ciertas cosas para poder atender porque la guía se quedaba corta... (F10: Trabajadora Social).

Desde el marco de Colombia, en términos generales, gran parte de la población tiene problemáticas de duelo... entonces es importante desde la intervención de Trabajo Social que los profesionales conozcamos un poco más a fondo sobre estrategias o metodologías a utilizar para abordar el tema de duelo. (E11: Trabajadora social).

Cuando las profesionales comprenden el duelo como el proceso siguiente a la muerte de un ser querido que requiere para su elaboración del cuerpo del fallecido, amarran el duelo a la existencia de pérdidas por muerte y dejan de lado otros tipos de pérdidas y los subsecuentes duelos ocasionados por el conflicto armado ${ }^{14}$. En la medida en

investigativo y de intervención en la formación del pregrado y posgrados de la Escuela, no solo desde el abordaje del dolor y el sufrimiento, sino desde distintas perspectivas disciplinares para la comprensión y la intervención de las problemáticas asociadas al contexto del conflicto armado colombiano.

13 La teoría como el punto de partida que soporta las maneras de aprehender y orientar el conocimiento de la realidad, otorgando el "valor y significado a la palabra como forma de comprender los hechos sociales" (Soto, 2012, p. 613) y de redimensionar la manera de responder a las situaciones que presentan las personas, incluye supuestos de carácter general que explican el funcionamiento de la sociedad y conceptos más específicos sobre temas concretos.

14 Según Ospina (2014) las pérdidas pueden clasificarse en pérdidas físicas o tangibles: pérdidas por muerte de seres queridos, pertenencias u otros objetos significativos, pérdidas simbólicas o psicosociales: representadas por cambios respecto al proyecto de vida, metas, relaciones, empleo, roles, entre otros. A su vez, las pérdidas parciales asociadas a avances o cambios en el desarrollo o relaciones del sujeto y las pérdidas totales, referidas a la muerte. En cuanto a las pérdidas ambiguas, son situaciones confusas en las que existe al mismo tiempo una presencia física y una ausencia psicológica, o viceversa, de una persona o relación, que requieren para su elaboración la resignificación de la ambigüedad existente. En el contexto del conflicto armado estas pérdidas se presentan de manera simultánea y se ven atravesadas por la subjetividad, historia y características personales de quienes lo vivencian. Para mayor profundidad se sugiere revisar el 
Escobar-Serrano, Charry-Higuera, y Ramírez-Moncada

que las profesionales no reconozcan la diversidad de pérdidas que trae el conflicto armado, no podrán escuchar, legitimar ni favorecer la tramitación del dolor y la resignificación de las pérdidas ambiguas, de la población con la que trabajan:

Para mí el duelo... yo siempre lo asociaba a una pérdida y no necesariamente social, sino una pérdida de una persona... (F10: Trabajadora Social).

Duelo: (igual) pérdida física, lo remitía solo a eso, duelo pérdida física, a veces al dolor (F4: Trabajadora social).

Ella aún no acepta que ese cuerpo que le entregaron sea el de su esposo, es un tema muy complejo porque tendrían que exhumar el cuerpo, hacer un proceso de ADN, certificar que efectivamente si sea el esposo, para que ella desde ahí pueda empezar su proceso de duelo... en ese caso no se pudo trabajar porque no se puede trabajar duelo sobre una persona que no se sabe dónde está, si está vivo o si está muerto. (E10: Trabajadora social).

En la intervención de las profesionales se evidencian cierta distancia, temor y resistencia a abordar el duelo, y cuando lo hacen parten de la premisa de que el duelo ya está superado. Sin embargo, los duelos generados en situaciones de violencia son complejos y sobrepasan las capacidades individuales de las personas, superando las posibilidades de adaptación, las diversas maneras de manifestación, trayendo alteraciones a la salud y daño al tejido social (Díaz, 2012; Uribe, 2008; Worden, 2009). No obstante, aunque la intervención profesional requiera articular la comprensión de aspectos de la psique humana que favorezcan la resolución de traumas y conflictos de separación en sus dimensiones individual, familiar y social, las profesionales enfatizan en una elaboración del duelo más individual y por tanto, justifican la intervención desde el área clínica o psicológica15,

Es que genera muchas dudas al momento de trabajar. Tengo otras compañeras que evitan a toda costa el tema, porque dicen que es un tema demasiado complejo y ellas prefieren ahí no meterse; yo creería que sí, pues partiendo de que ya está superado. (E10: Trabajadora social).

Los remito a psicólogo porque yo ya no puedo seguir con ellos, es muy complejo, la verdad. (E10: Trabajadora social).

El duelo, como lo decía ahora, cada persona lo asume de diferentes formas, y para mí es muy importante siempre resignificar eso que la persona está sintiendo en términos

Trabajo de Grado: Procesos de duelo de personas que vivenciaron el desplazamiento forzado en el marco del conflicto armado colombiano de Ramírez y Trujillo (2019), trabajo que hizo parte de la investigación en curso.

15 Para el Trabajo Social, hacer de su práctica reflexiva un campo de conocimiento sobre la intervención, implica acudir a su formación transdisciplinaria y ampliar el término de lo clínico, no referido a intervenciones netamente psicológicas, médicas, psicoanalíticas o psiquiátricas, sino más a un modo de analizar, comprender y actuar del profesional bajo ciertas lógicas de representación que deben reconocerse. Este tipo de acercamientos justifican la importancia de mantener el debate de la transdisciplinariedad en la intervención de problemáticas sociales (Karsz, 2007). 
generales, que logre identificar que hay un dolor que está ahí, que tengo que aceptarlo, pero que de cierta forma también tengo que dejarlo ir, por decirlo así, dependiendo de la capacidad de cada persona, en su tiempo. (E11: Trabajadora social).

Otra de las características que reconocen las profesionales es que en el contexto del conflicto armado la muerte es demasiado dolorosa y silenciosa por lo cual se deja de lado y no se habla. Según Ospina (2014) y Worden (2009) en contextos de violencia, el silencio es necesario para subsistir y sobrevivir, sin embargo, estos duelos se posponen y se enmascaran, convirtiéndose en fuentes de sufrimiento físico y emocional. Los duelos pospuestos se refieren al aplazamiento de las principales tareas del duelo relacionadas con el aceptar y el experimentar el dolor de las pérdidas, debido a que al mismo tiempo en que ocurren las pérdidas, los sobrevivientes resultan amenazados en su supervivencia y/o han tenido que huir imposibilitando la realización de los rituales sociales y familiares necesarios para avanzar en la elaboración de los duelos. Los duelos enmascarados, se caracterizan por débiles reacciones y expresiones de dolor y somatizaciones ya que no pueden reconocerse abiertamente las pérdidas debido a distintas situaciones sociopolíticas.

La intervención profesional se evita o se diluye, al normalizar las dificultades de elaboración de estos duelos, como con la momificación o conservación de las pertenencias que los muertos poseyeron de la misma manera que cuando estaban vivos, y la somatización relacionada con la represión de la información y del dolor familiar. El silencio puede comprenderse como un límite comprensivo entre la palabra y la posibilidad de otorgar sentido y significado a las experiencias. En cuanto al dolor, el silencio evoca la dificultad de nombrar lo innombrable, al tiempo que invita a una elaboración metafórica a través de caminos distintos al de la palabra (Garriga, 2013; Le Breton, 2009):

Ella lo entierra, pero igual sigue con la duda de si ese cuerpo es el de su esposo o no, eso no ha permitido que ella salga todavía del dolor, que todavía en algunos momentos lo sienta, con mucha facilidad... a partir de eso ella perdió la movilidad del brazo. La dinámica familiar cambió totalmente, se empezaron a manejar un poco de agendas ocultas y demás, a partir de todo el tema del conflicto armado y de los asesinatos, y esa desaparición. En esos temas de duelo hay muchas cositas que se quedan como en el aire y prefiere la familia como no contarla. La señora aún sufre por la pérdida de su esposo, no duerme, debido a eso se, empezó a guardarse todos esos sentimientos y se generó una fibromialgia y una fibrosis. La señora aún tiene la ropa de su hijo ahí en la casa, la tiene ahí tal cual como él la dejó, escucha las canciones que a él le gustaban, no permite que nadie en la casa hable de él, o sea, el tema en la casa es sagrado, nadie puede decir absolutamente, ni siquiera pueden mencionar el nombre, pero cada vez que llegan las fechas especiales o llegan los cumpleaños o demás, la señora lo recuerda como, pues, como si estuviera vivo. (E10: Trabajadora social). 
Escobar-Serrano, Charry-Higuera, y Ramírez-Moncada

El conflicto armado los deja tan marcados que a ellos les da temor hablar de eso, porque sucede que a pesar de que ellos se desplazan a otra ciudad, llegan y se encuentran con grupos muy similares a los que estaban en la costa, o en el pueblo en el que ellos estaban...grupos al margen de la ley, que de cierta forma cohíben que esa persona hable o exprese lo que vivieron donde estaban. (E11: Trabajadora social).

Para las profesionales, las familias presentan múltiples problemas ocasionados por sus duelos en el contexto del conflicto armado, los cuales se vislumbran en términos de tiempo, reacciones y posibilidades de intervención:

Cuando uno llega a las familias encuentran múltiples problemas, uno piensa que solamente voy a articular o a mejorar la comunicación, límites de crianza, uno se enmarca como en unos lineamientos básicos... en la tercera o cuarta sesión de roles me di cuenta que no era roles, si no efectivamente duelo, porque a la señora el esposo se le desapareció y días después lo encontraron muerto, desde ahí vienen los problemas, no tanto de roles. Desde ahí ya se comienza a dificultar un poco la intervención... (E11: Trabajadora social).

\subsection{Historia de pérdidas del profesional y la relación con la intervención en duelo}

La autorreferencia es entendida como la práctica reflexiva de observarse a sí mismo frente a la presencia de otro que da cuenta del reconocimiento de que nuestra historia personal siempre está presente y que es imposible separar "lo que se es" de "lo que se describe" (Garzón, 2008). Sánchez (2014) desarrolla el concepto de heterorreferencia, para describir una acción que favorece la observación y reflexión con los otros de los puntos ciegos, cognitivos y emocionales que todos los seres humanos tenemos y que no nos permiten vernos a nosotros mismos. En el relato de las profesionales se destaca la reflexión acerca del reconocimiento y/o elaboración de su historia de pérdidas y la afectación en la intervención en duelo con la población.

De un lado, el relato de su historia de pérdidas las remite a hablar sobre la intervención en duelo con la población, desde su propia experiencia de elaboración, siendo un referente para el proceso de intervención:

Identificar con ellos las potencialidades y recursos de afrontamiento que tienen desde sus necesidades, entonces ahí siento que de pronto emocionalmente me vinculaba porque yo llegaba y reflexionaba, porque yo también soy víctima del conflicto armado pero... yo canalicé de cierta manera diferente, yo me gradué de mi universidad, yo potencialicé mis habilidades. Yo, como que, les decía que buscáramos los recursos, que pensáramos entre esa familia y, pues, yo, qué podíamos hacer para de pronto salir de esa situación (E5: Trabajadora social). 
Escobar-Serrano, Charry-Higuera, y Ramírez-Moncada

De otro lado, el relato de la intervención en duelo que realizan de los casos moviliza el proceso de reflexión de su historia de pérdidas:

Por lo que movió inicialmente, me puso a pensar muchas cosas a nivel personal en tanto que yo decía: bueno, yo soy trabajadora social y estoy para servir a muchos, pero entonces cómo estoy yo. Entonces empecé a mirar que quizás había situaciones en las que uno, a veces, es muy bueno dando consejos, organizando ciertas cosas o empoderando a ciertas personas para que puedan salir de donde están, pero en qué momento uno lo puede aplicar hacía la vida de uno. Se hace más complejo reconocer cosas. (F10: Trabajadora social).

Para otra profesional, la identificación con personas que han tenido pérdidas en similares circunstancias a la suya la bloquea emocionalmente, generando resistencia o malestar en la intervención, que al mismo tiempo moviliza la necesidad de trabajar sobre la elaboración de las propias pérdidas:

Justamente, la forma como se llevan al esposo y todo eso era muy parecida a la forma como asesinaron a mi papá. Es muy complejo, la verdad, yo a veces llegaba a la oficina o llegaba a la casa y yo "¿qué voy a hacer con esa comunidad?", "qué voy a hacer con ella". Pues la señora sí estaba como muy dispuesta a querer trabajar el tema del duelo, pero yo no, yo no podía trabajar. Entonces cuando llegaba a la casa o llegaba nuevamente a la oficina se me venían como reflejos y empezaba a hacer como el proceso de comparación... El duelo de mi papá yo le hice muy rápido, pero no sé si de pronto al empezar a trabajar cosas se vayan a remover algunas situaciones que yo considero que ya las pude superar. No sabré decirte, pero pues para mí en estos momentos, ya hablo con facilidad del tema. Anteriormente no se podía hablar del tema para nada, era más bien, no era como tristeza, sino como una rabia que me invadía totalmente, entonces pues no, ahora pues ya es con mucha facilidad, ya hablo del tema y no me genera ningún tipo de emoción, entonces pues yo creería que para mí ya el duelo pasó. (E10: Trabajadora social).

Por último, los relatos de las profesionales exponen procesos autorreferenciales a partir de las provocaciones reflexivas en el marco del proyecto de investigación:

Yo siento que como te sientes tú puedes aportar a otras personas, entonces ya como que uno escribiendo que es el dolor, que es el miedo, que es realmente un duelo, teniendo claridad en ciertas cosas a uno no le va a dar miedo trabajarlo con otras personas. (F4: Trabajadora social).

Me puso a pensar muchas cosas a nivel personal. Yo decía: bueno, yo soy trabajadora social y estoy para servir a muchos, pero entonces cómo estoy yo, (...) me hizo reconocer como que habían duelos, pérdidas que aún no se habían superado, cosas que yo pensé que estaban muy claras en mí, me di cuenta que no, entonces eso fue lo que me hizo continuar, hacer el esfuerzo de venir el miércoles, me hizo seguir como en ese espejo de que probablemente al terminar el curso podía terminar de resolver ciertas cositas y generar más estrategias para poder reconocer ciertas cosas en mí y aportar a 
Escobar-Serrano, Charry-Higuera, y Ramírez-Moncada

las personas que posiblemente vaya a atender. La verdad es que me sirvió mucho, personalmente llegó un punto en el que llegué a la casa a hablar con mi hermano, porque mi papá se murió. Los primeros días se habló del tema y después no más, entonces como que volvimos a hablar del tema, con mucha más facilidad. (F10: Trabajadora social).

Es lo que Velázquez (2012) reconoce en el trabajo en contextos de violencia como la necesidad de no sólo conocer sobre la temática que se está abordando, sino también "saber sobre sí en relación a esos efectos" (p. 140), articulando los conocimientos y experiencias con la observación de las reacciones propias.

Estas reflexiones son abordadas ampliamente por Worden (2009) quien sostiene que el profesional es afectado y afecta la intervención en duelo. Trabajar con personas en duelo nos hace conscientes de nuestras pérdidas y duelos, sobre todo cuando hay similares circunstancias; la posibilidad de resolver tales pérdidas tendrá un lugar a la hora de intervenir con los demás, ya sea como un enganche que favorezca o como un bloqueo que obstaculice el establecimiento de la relación profesional y el alcance de la intervención. Por lo tanto, la intervención en duelo puede constituirse en una práctica desgastadora emocional y ontológicamente, que debe ser comprendida y manejada por las profesionales:

Estas emociones están íntimamente ligadas a distintos procesos de reflexividad. El dolor del otro o la otra despierta en ocasiones estados emocionales hasta entonces desconocidos por las intervinientes. Las emociones que generan las personas desplazadas -y las múltiples pérdidas a las que se enfrentan- movilizan a las intervinientes y explican en buena medida tanto el grado de compromiso profesional que desarrollan con esta población como el tipo de acompañamiento que le brindan a cada persona, grupo, familia o pueblo étnico. (Mosquera, 2012, p. 18).

El permitir un espacio de reflexión donde se le diera palabra a la diversidad de sentimientos suscitados en las profesionales, favoreció el reconocimiento de sus procesos de pérdidas y la conexión con la intervención en duelo, siendo transversal la comprensión teórica de lo que se entiende como duelo, "las emociones experimentadas por las intervinientes se convierten en recursos clave a la hora de entender cómo se producen los saberes de acción interventivos" (Mosquera, 2012, p. 18).

\section{Conclusiones}

La reflexividad representa para el profesional una oportunidad de pensar sobre lo que hace, cómo lo hace, para qué lo hace y el lugar que asume en este hacer. Es decir, debe ser una posibilidad para reconocer las relaciones de poder, los constreñimientos emocionales y las dominaciones sociales, políticas y económicas que gobiernan la acción profesional.

\footnotetext{
Prospectiva. Revista de Trabajo Social e intervención social • No. 30• jul.-dic. 2020 • pp. 67-89 e-ISSN: 2389-993X • https:/ / doi.org/10.25100/ prts.v0i30.8564
} 
Retomar el sentido de la intervención profesional en duelo del Trabajo Social, en el caso del programa PAPSIVI, inicia por reconocer las limitaciones y las tensiones entre los lineamientos y su implementación en la cotidianidad de la población y de los profesionales. Convoca asuntos ontológicos, epistemológicos y políticos, por ejemplo, en la reiterada diferencia que hacen las profesionales entre intervención y atención, ya que tal nominación remite a una subjetivación distinta del profesional que asume la vinculación con las personas afectadas por el conflicto armado. En tal distinción se aprecia una preocupación que trasciende tensiones nominativas, al constreñimiento de la intervención como un servicio, acorde a protocolos y reglamentaciones jurídicas, que deja de lado aspectos fundantes de la intervención como proceso social: el lugar del profesional que asume la intervención, la relación con las personas con las que trabaja, el lugar del tiempo en los procesos de cambio y transformación social, las relaciones de poder institucional, el reconocimiento de las singularidades y realidad de las personas con las que se interviene, así como los efectos subjetivos que produce en el profesional el trabajo con el dolor y el sufrimiento humano. La revisión de la historia de pérdidas del profesional es útil para ampliar el alcance y profundidad de la intervención en duelo y favorecer el cuidado emocional de los profesionales que atienden población afectada por el conflicto armado. Sin embargo, los procesos de intervención en duelo en el marco del conflicto armado exigen al profesional no sólo prepararse emocionalmente en torno de sus propias pérdidas, sino formarse psicológica, sociológica, antropológica y políticamente.

\section{Referencias bibliográficas}

Bello, M. (2009). Los daños y las pérdidas asociadas al desplazamiento forzado en las ciudades. Revista Ciudad Pazando, 2(1), 190-202. doi: 10.14483/2422278X.7390.

Benyakar, M. (2016). Lo disruptivo y lo traumático. Abordajes posibles frente a situaciones de crisis individuales y colectivas. Argentina: Universidad Nacional de San Luis. Recuperado de http:/ / www.neu.unsl.edu.ar/wp-content/uploads/2018/03/Discruptivotraumatico.pdf.

Betancourt, L. (2011). Impactos psicosociales de la violencia política. En L. Betancourt, C. Perdomo, A.N. Rodríguez y C. Castro, Entre la violencia, la no violencia y la construcción de poder (pp. 77-96). Cali: Programa Editorial Universidad del Valle.

Blandón, T., Durango, S., y Palacio, V. (2017). La labor del trabajador social con familias en situación de desplazamiento forzado en el sector poblado la calle del municipio de Girardota durante el primer semestre del año 2017 (Tesis de pregrado). Corporación universitaria Minuto de Dios, Bello, Colombia. Recuperado de https:/ / repository.uniminuto.edu/bitstream/handle/10656/5234/TTS_BlandonOs orioTaniaLizeth_2017.pdf?sequence $=1 \&$ isAllowed $=y$.

Bourdieu, P., y Wacquant L. (2005). Una invitación a la sociología reflexiva. Argentina: Siglo Veintiuno. 
Escobar-Serrano, Charry-Higuera, y Ramírez-Moncada

Cancimance-López, A. (2013). Memoria y violencia política en Colombia. Los marcos sociales y políticos de los procesos de reconstrucción de memoria histórica en el país. Eleuthera, 9(2), 13-38.

Cancimance-López, A. (2015). Los silencios como práctica de resistencia cotidiana: narrativas de los pobladores de El Tigre, Putumayo, que sobrevivieron al control armado del Bloque Sur de las AUC. Boletín de Antropología, 30(49), 137-159.

Carbonell, O.A., Plata, S.J., Bermúdez, M.E., Suárez, L.C., Peña, P.A., y Villanueva, C. (2015). Caracterización de prácticas de cuidado en familias colombianas con niños en primera infancia en situación de desplazamiento forzado. Universitas Psychologica, 14(1), 67-80.

Casillas, O.L. (2018). Intervención del sufrimiento, relaciones y estrés traumático secundario en trabajadores sociales. Revista de Trabajo Social, 20(1), 103-130. doi: $10.15446 /$ ts.v20n1.71564.

Chaurand, A., Feixas, G., y Neimeyer, R. (2010). El inventario de Historia de Pérdidas (IHP): presentación y utilidad clínica. Revista Psicoterapia, 21(84), 95-101. Recuperado de http:/ / diposit.ub.edu/dspace/ bitstream/2445/65387/1/600533.pdf.

Chávez, Y.A., y Bohórquez, M.C. (2011). Desplazamiento forzado y reconfiguraciones familiares: una mirada a la situación de madres solteras adolescentes en Soacha. Prospectiva. Revista de Trabajo Social e Intervención social, (16), 289-304.

CICR. (2016). No los olvidamos. Necesidades de los familiares de las personas desaparecidas en Colombia. Evaluación realizada por el Comité Internacional de la Cruz Roja Dirigido a las entidades estatales con incidencia en la temática de la desaparición y la atención a familiares de personas desaparecidas y al público en general. Bogotá: CIRC; Desaparecidos. Recuperado de https:/ / www.refworld.org.es/pdfid/5a70e4244.pdf.

Congreso de Colombia. (10 de junio de 2011). Ley de víctimas. [Ley 1448 de 2011]. DO: 48.096

Di Maio, L. (2015). El tema del duelo en la práctica del trabajador social. Trabajo Social, (17), 239-252.

Díaz, V.E. (2012). Del duelo individual a la dimensión social del duelo en contextos de violencia. En O.E. Navarro-Carrascal (Comp.), Psicología social: temas, teorías y aplicaciones (pp. 187-204). Colombia: Universidad de Antioquia.

Díaz, V.E., Molina, A.N. y Marín, M.A. (2015). Las pérdidas y los duelos en personas afectadas por el desplazamiento forzado. Pensamiento Psicológico, 13(1), 65-80.

Dubet, F. (2010). Sociología de la experiencia. España: Editorial Complutense.

Fombuena, J. (2011). Un estudio de las familias de origen de los y las trabajadoras sociales desde el modelo contextual. Comunitania, (2), 23-37. Recuperado de http:/ / revistas.uned.es/index.php/comunitania/article/view/7105.

García, M. (2013). El proceso de duelo en psicoterapia de tiempo limitado, evaluado mediante el método del tema central de conflicto relacional (CCRT) (Tesis doctoral). Facultad de Filosofía, Universidad Complutense de Madrid. Madrid, España. 
Escobar-Serrano, Charry-Higuera, y Ramírez-Moncada

García, S., y Rendueles, C. (2017). Hacia un nuevo Trabajo Social crítico: el gobierno de lo social en la era neoliberal. Presentación del Monográfico. Cuadernos de Trabajo Social, 30(2), 243-260. doi: 10.5209/CUTS.56352.

Garriga, R. (2013). El silencio como límite comprensivo: una aproximación a su aplicación en las propuestas artísticas de Shimon Attie y Alfredo Jaar que emanan del eco de acontecimientos bélicos. Eikasia. Revista de filosofía, (50), 323-334.

Garzón, D.I. (2008). Autorreferencia y estilo terapéutico: su intersección en la formación de terapeutas sistémicos. Diversitas, 4(1), 159-171.

Gledhill, J. (2000). Antropología y política: compromiso, responsabilidad y ámbito académico. En J. Gledhill, El poder y sus disfraces: perspectivas antropológicas de la política (pp. 337-383). Barcelona: Bellaterra.

Gómez, N., y Rodríguez, A. (2017). Intervención de trabajo Social en duelo con personas diagnosticadas con cáncer en Cali: trayectorias, discursos, intencionalidades y reflexividades de dos trabajadoras sociales (Tesis de pregrado). Universidad del Valle. Cali, Colombia.

González, F. (2014). Poder y violencia en Colombia. Bogotá: Odecofi; Cinep; Colciencias.

GOV.CO. (2018). Registro Único de Víctimas en Colombia. Unidad Para la Atención y Reparación Integral de Víctimas. Recuperado de https://www.unidadvictimas.gov.co/es.

Guzmán, G., Fals-Borda, O., y Umaña, E. (1962). La violencia en Colombia. Estudio de un Proceso social. Tomo I. Bogotá, Colombia: Ediciones Tercer Mundo.

Ibáñez, J. (1994). El regreso del sujeto la investigación social de segundo orden. España: Siglo Veintiuno Editores.

Karsz, S. (2007). Problematizar el trabajo social. Definición, figuras, clínica. Barcelona, España: Editorial Gedisa.

Lasso, P. (2013). Cuando se vive el desarraigo. Educación y desplazamiento forzado: una mirada desde el Distrito de Aguablanca, Cali, Colombia. Revista Guillermo de Ockham, 11(2), 35-51. doi: $10.21500 / 22563202.608$.

Le Breton, D. (2009). El silencio. Aproximaciones. Madrid: Sequitur.

Meza, J.L, (2012). Aprender el cuidado del otro: una urgencia en la formación moral de un país en el cual nos estamos matando. Actualidades Pedagógicas, (60), 215-235.

Montagud, X. (2015). Complejidad, reflexividad y autoetnografía. Las posibilidades de la investigación narrativa en la mejora de la práctica profesional. Trabajo Social Global, 5(9), 3-23

Moreno, M.A. y Díaz, M.E. (2016). Posturas en la atención psicosocial a víctimas del conflicto armado en Colombia. El Ágora, 16(1), 193-213. Recuperado de https://revistas.usb.edu.co/index.php/Agora/article/view/2172/1900.

Moreno, M.A., y Moncayo, J.E. (2015). Consideraciones conceptuales y alternativas de análisis en el escenario de atención a víctimas del conflicto armado. En J.E. Moncayo y A. Díaz (Eds.), Psicología social crítica e intervención psicosocial. Reflexiones y experiencias de investigación (pp. 37-56). Cali: Editorial Universidad de San Buenaventura. 
Escobar-Serrano, Charry-Higuera, y Ramírez-Moncada

Mosquera, C. (2006). Conocimiento científico y saberes de acción en Trabajo Social: valoraciones, desconocimientos y revaloraciones una lectura desde los países de América del Norte. Trabajo Social, (8), 131-142.

Mosquera, C. (2012). Las prácticas de las intervinientes en los procesos de atención psicosocial a la población desplazada por la violencia sociopolítica Colombiana. Trabajo Social, (14), 11-27.

Munévar, M., y Castro, X. (2018). Escuchando a las víctimas del conflicto armado colombiano: la experiencia de un dispositivo de atención psicosocial. Revista CS, (25), 81-109. doi: $10.18046 /$ recs.i25.2220.

Neimeyer, R. (2002). Aprender de la pérdida. Una guía para afrontar el duelo. Barcelona: Editorial Paidós.

Oficina de Promoción Social. (2018a). Orientaciones metodológicas para la atención familiar. Bogotá: Oficina de Promoción Social.

Oficina de Promoción Social. (2018b). Orientaciones metodológicas para la atención individual. Bogotá: Oficina de Promoción Social.

Ospina, A. (2014). Cuando muere un ser amado. Cómo comprender y afrontar el duelo por muerte. Cali, Colombia: Programa Editorial Universidad del Valle.

Páramo, P. (2013). La construcción Social del Conocimiento Científico En P. Páramo (Comp.), La investigación en ciencias sociales: discusiones epistemológicas (pp. 183-194). Bogotá: Universidad Piloto de Colombia.

Peláez, G. (2007). Los duelos en el cuerpo físico y social de mujeres víctimas de la violencia. Antípoda. Revista de antropología y arqueología, (5), 75-95.

Portilla, A.C., y Correa, C. (2015). Estudio sobre la implementación del Programa de Reparación Individual en Colombia. Recuperado de https://www.ictj.org/sites/default/files/ICTJCOL-Estudio-reparacion-individual-2015.pdf.

Ramírez, N., y Trujillo, V. (2019). Procesos de duelo de personas que vivenciaron el desplazamiento forzado en el marco del conflicto armado colombiano (Tesis de pregrado). Universidad del Valle. Cali, Colombia.

Riaño, P. (1998). Recuperar las memorias y elaborar los duelos. En I. Cepeda y C. Girón, Duelo, memoria, reparación (pp. 103-117). Colombia: Fundación Manuel Cepeda Vargas; Defensoría del Pueblo.

Rodríguez, M.R. (2017). Tres mujeres desplazadas por la violencia, reubicadas en Cali, resignifican sus experiencias como ciudadanas entre los años 2005 y 2017. Estudios de caso (Tesis de maestría). Instituto de Educación y Pedagogía. Universidad del Valle.

Sánchez, L.M. (2014). Auto y heterorreferencia: intervención, supervisión. Trabajo Social, (16), 127-141.

Recuperado

de https:/ / revistas.unal.edu.co/index.php/tsocial/article/view/47063.

Sánchez, L.M., y Escobar, M.C. (2009). Mitos y Secretos Familiares. Cali: Programa Editorial Universidad del Valle. 
Escobar-Serrano, Charry-Higuera, y Ramírez-Moncada

Soto, C. (2012). La migración internacional paterna o materna: una lectura desde los sujetos jóvenes. Revista Latinoamericana de Ciencias Sociales, Niñez y Juventud, 68(2), 141-166.

Torres, A. (2004). Por una investigación desde el margen. En A. Jiménez y A. Torres (Comps.), La práctica investigativa en ciencias sociales (pp. 62-79). Bogotá: Universidad Pedagógica Nacional.

Torres, A., y Torres, J. (2000). Subjetividad y sujetos sociales en la obra de Hugo Zemelman. Revista Folios, (12), 1-18.

Torres, N. (2011). Desplazamiento forzado y enfoque diferencial.Una posibilidad de intervención / Acción en ámbitos de exclusión. Tendencias, 12(2), 106-122. Recuperado de http://revistas.udenar.edu.co/index.php/rtend/article/view/537/587.

Uribe, M.T. (2008). Los duelos colectivos: entre la memoria y la reparación. Agenda Cultural Alma mater, (149), 1-11.

Vargas, J. (2009). Desafíos ético-políticos para las y los profesionales de Trabajo Social en contextos de conflicto armado y desplazamiento forzado. El caso Colombiano. Recuperado de http:// www.ts.ucr.ac.cr/binarios/congresos/reg/slets/slets-019-112.pdf.

Velázquez, S. (2012). Violencias y familias. Implicancias del trabajo profesional: el cuidado de quienes cuidan. Paidós. Argentina.

Worden, W. (2009). El tratamiento del duelo: asesoramiento psicológico y terapia. España: Ediciones Paidós. 


\section{OTROS ARTÍCULOS DE PROSPECTIVA No. 30 DE 2020}

\section{EDITORIAL}

Coherencia, integridad y vida cotidiana

Luz Mary Sánchez-Rengifo

\section{ARTÍCULOS}

Trabajo Social y medios de comunicación: perspectivas y posibilidades de encuentro Social

Mercedes Muriel-Saiz

Maribel Martín-Estalayo

Seguridad, afectos y familias. Obstáculos en el proceso de reintegración de personas desmovilizadas de grupos

armados en Santander, Colombia

Jakeline Vargas-Parra

Ángela María Díaz-Pérez

Priscyll Anctil-Avoine

Reflexividad sobre la intervención profesional en duelo con población afectada por el conflicto armado en Colombia

María Cénide Escobar-Serrano

Maritza Charry-Higuera

Natalia Ramírez-Moncada

Ser mujer indígena, náhuatl, casada, migrante, sin trabajo remunerado: una realidad en los albergues jornaleros agrícolas en Colima, México

Nancy Elizabeth Molina-Rodríguez

Tipos y manifestaciones de la violencia de género: una visibilización a partir de relatos de mujeres víctimas en Soacha, Colombia

Diana Carolina Tibaná-Ríos

Diana Alejandra Arciniegas-Ramírez

Ingrid Julieth Delgado-Hernández

Análisis de necesidades en familias monoparentales con jefatura femenina usuarias de servicios sociales de atención primaria en España

Celia María Fernández-Martínez

Manuela Avilés-Hernández

Experiencias de intervención de trabajadoras sociales con trabajadores sexuales masculinos en Bogotá, Colombia Johan Arturo Barrera-Castellanos
Condiciones laborales de trabajadores sociales en hospitales públicos en la provincia de Mendoza,

Argentina

Cecilia Amalia Molina

Yanina Noemi Roslan-Angeloni

Analía Graciela Correa

Viviana Elena Varela

Gubernamentalidad neoliberal: miradas desde las intervenciones del Trabajo Social en el Gran La Plata, Argentina

Paula Mara Danel

Marcela Claudia Velurtas

Agustina María Favero-Avico

Educación superior en Trabajo Social en Chile y formación para la intervención en situación de calle. Desafios desde la evaluación que interventores hacen de sus procesos de práctica pre-profesional

Carlos Alejandro Andrade-Guzmán

Ignacio Andrés Eissmann-Araya

Educación en Derechos Humanos para el Trabajo Social en Chile: una mirada desde los estándares

internacionales

Lury Soledad Reyes-Pérez

Vivianne Soledad Hasse-Riquelme

Luis Marcelo Silva-Burgos

Arriesgar y preservar la vida: derechos humanos, conflicto sociopolítico armado y Trabajo Social en Colombia

Cristian Sebastián Castaño-Orozco

Ricardo Patiño-Martínez

\section{IN MEMORIAM}

Cristina Bautista Taquinás. Mujer indígena Nasa, Trabajadora Social, lideresa del norte del Cauca, Colombia

Alba Nubia Rodríguez-Pizarro

Lady Johanna Betancourt-Maldonado
ARTISTA INVITADO
MALA JUNTA KLAN
Alejandra Gutiérrez-Cárdenas

\section{PROSPECTIVA}

